Check for updates

Cite this: Chem. Sci., 2019, 10, 7788

๑ All publication charges for this article have been paid for by the Royal Society of Chemistry

Received 28th May 2019

Accepted 2nd July 2019

DOI: $10.1039 / c 9 s c 02572 c$

rsc.li/chemical-science

\section{Bio-inspired synthesis of xishacorenes A, B, and C, and a new congener from fuscol $\dagger$}

\author{
Alexander R. Rovira, (D) ${ }^{a}$ Nicolas Müller, (D) ${ }^{a}$ Weiwen Deng, ${ }^{b}$ Chudi Ndubaku \\ and Richmond Sarpong (D) *a
}

\begin{abstract}
The xishacorene natural products are structurally unique apolar diterpenoids that feature a bicyclo[3.3.1] framework. These secondary metabolites likely arise from the well-studied, structurally related diterpenoid fuscol. In this manuscript, we describe the conversion of fuscol to xishacorenes A, B, and C, as well as a previously unreported congener, which we have named xishacorene D. In addition, we describe immunomodulatory activity studies of the xishacorenes, a structurally related analogue, and fuscol. These studies were aided by an accurate determination of the physical properties (e.g., molar extinction coefficient) of the highly apolar xishacorenes.
\end{abstract}

\section{Introduction}

Polyene cyclizations are among the most powerful methods for the rapid construction of $\mathrm{C}-\mathrm{C}$ bonds in carbon-based frameworks. These cyclizations, which appear in the biosyntheses of a wide array of topologically complex natural products, often proceed through concerted, stereocontrolled pathways. ${ }^{1}$ Of the natural products formed through polyene cyclizations, terpenederived secondary metabolites (terpenoids) are among the most studied. While the biosynthetic pathways that lead to many terpenoid molecules have formed the basis for their chemical syntheses, synthetic endeavors toward terpenoids that exploit polyene cyclizations can provide insights into the role of these reactions in their biosynthesis as well. ${ }^{2}$

The xishacorene diterpenoids (see 1-3, Fig. 1), were isolated in 2017 from the soft coral Sinularia polydactyla off the coast of the Xisha Islands in the South China Sea. ${ }^{3}$ These secondary metabolites were obtained alongside a structurally-related terpenoid, fuscol (5), from which 1-3 are proposed to arise through an acid-mediated polyene cyclization (Fig. 1). ${ }^{3}$ Fuscol was first isolated in 1978 by Schmitz and coworkers. ${ }^{4}$ In considering the biogenesis of the xishacorenes, fuscol appears to be the likely progenitor. However, the link between these natural products has not been realized in a laboratory setting. ${ }^{5}$ Furthermore, as outlined by the cyclization sequence illustrated in Fig. 1, one would have expected the isolation of compound 4, a diastereomer of $\mathbf{3}$, which we have named "xishacorene D", alongside the other xishacorene congeners.

${ }^{a}$ Department of Chemistry, University of California, Berkeley, California 94720, USA. E-mail: rsarpong@berkeley.edu

${ }^{b}$ Aduro Biotech, Inc., 740 Heinz Ave, Berkeley, California 94720, USA

$\dagger$ Electronic supplementary information (ESI) available. See DOI: $10.1039 / \mathrm{c} 9 \mathrm{sc} 02572 \mathrm{c}$
Evidence that the xishacorenes can arise from fuscol would provide key support for a unified biosynthesis of these natural products from geranylgeranyl pyrophosphate (GGPP, 8), as it has been shown that 5 can arise from eunicol (6) through a Cope rearrangement. ${ }^{6}$

Fuscol and its glycosylated derivatives (e.g., fuscoside B, 8, Fig. 2A) possess significant anti-inflammatory activity. ${ }^{7}$ Given their putative biosynthetic connections, analogous activity might be anticipated for the xishacorenes. A preliminary biological screening of 1-3 by Guo et al. revealed that these molecules possess notable activity as promoters of concavalin A (ConA)-induced T-lymphocyte proliferation. ${ }^{3}$ This observed activity supports a possible link between fuscol and the xishacorenes as immune modulators. ${ }^{8}$ However, the capacity of the xishacorenes to act as antagonists of immunological pathways has not previously been studied. ${ }^{9}$

In 2018, we reported the first total synthesis of xishacorene B from $R-(-)$-carvone as part of a program aimed at the synthesis of structurally complex molecules from inexpensive "chiral pool” precursors. ${ }^{\mathbf{1 0 , 1 1}}$ While our synthetic plan provided access to 2 , it was not easily extended to the syntheses of the other xishacorenes, especially 3, which possesses an exo methylene group that would be difficult to install using our previously reported approach. It has been recognized that fuscol, which has been synthesized by several groups, ${ }^{12}$ could serve as a precursor to all xishacorene congeners. Access to the xishacorenes from fuscol would not only provide support for their proposed biosynthesis, but also facilitate further investigations into their bioactivity.

Herein, we report a preparation of the known xishacorenes (i.e., A-C; 1-3) and the previously unreported congener xishacorene D (4), along with a fuscol-like analog (10, Fig. 2B). Furthermore, we provide revised physical data for the xishacorene natural products and biological assays of 1-5 and $\mathbf{1 0 .}$ 


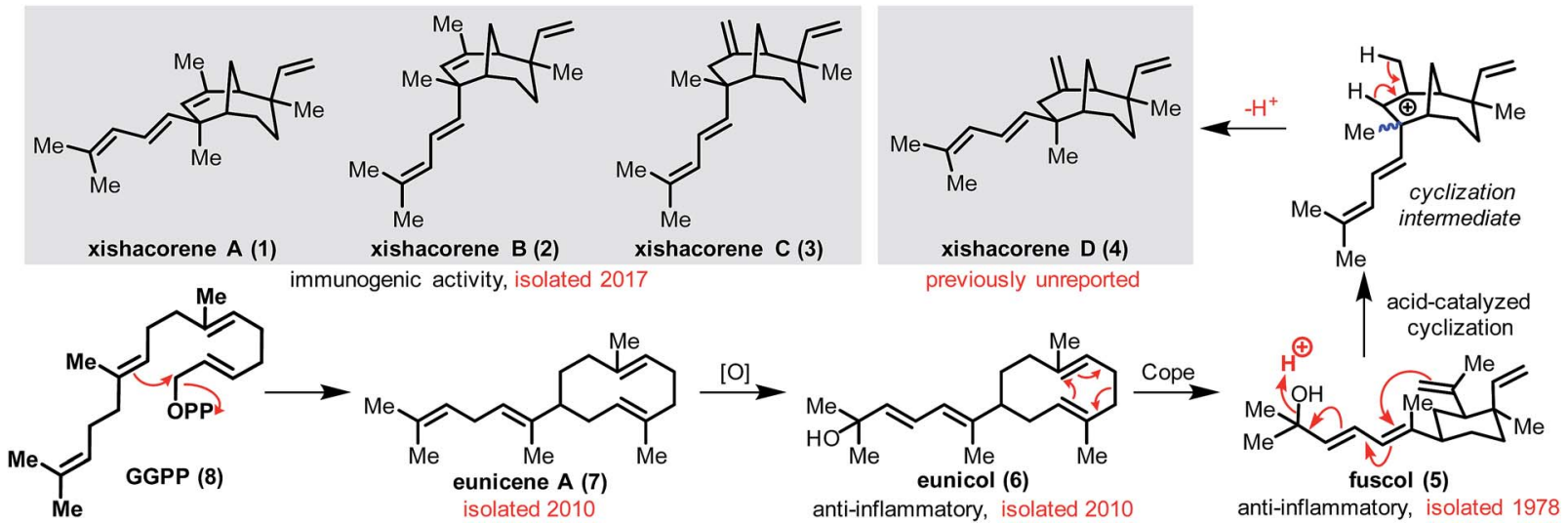

Fig. 1 Proposed biosynthesis of the xishacorene family from GGPP.

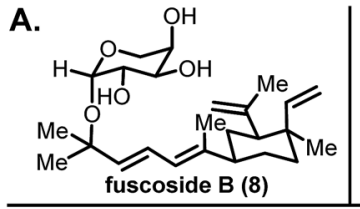

B.
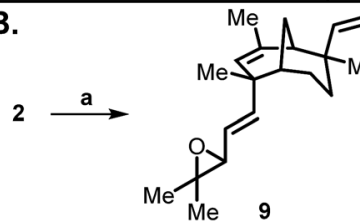
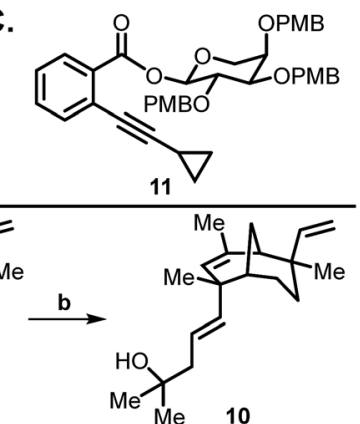

Fig. 2 (A) Structure of fuscoside B; (B) Fuscol analogue 10 synthesis; (C) Alkyne-arabinose 11. Conditions: (a) $\mathrm{m}$-CPBA, DCM, $0{ }^{\circ} \mathrm{C}, 2 \mathrm{~h}(80 \%$ yield); (b) $\mathrm{LiAlH}_{4}, \mathrm{THF}, 0{ }^{\circ} \mathrm{C}, 4 \mathrm{~h}$ (87\% yield).

\section{Results and discussion}

\section{Synthesis of analogues of the xishacorenes}

Our studies that led to the preparation of all the xishacorenes commenced with an initial goal of obtaining a library of glycosylated derivatives of fuscol and xishacorene B for biological screenings. For this purpose, we synthesized a hydroxylated xishacorene B-derived analogue of fuscol (10, Fig. 2B) starting from 2, which we had previously prepared. ${ }^{11}$ Epoxidation of 2 with $m$-CPBA gave epoxide $\mathbf{9}$ as a mixture of diastereomers, which upon reduction with $\mathrm{LiAlH}_{4}$ yielded tertiary alcohol $\mathbf{1 0}$ (70\% yield over two steps). With $\mathbf{1 0}$ in hand, we then sought to prepare fuscoside B (8), which would enable us to test the glycosylation of these tertiary alcohols including $\mathbf{1 0}$. While there have been several attempts to prepare the fuscosides, ${ }^{13}$ only the undesired $\beta$-anomer of $\mathbf{8}$ has been synthesized in low yield. We posited that modern glycosylation strategies, such as those developed by $\mathrm{Yu}$ et al. ${ }^{14}$ using gold catalysis with an alkyne glycoside donor (e.g. 11, Fig. 2C), would enable mild, relatively neutral glycosylation conditions to access $8 .^{15}$ The fuscol (5) required for these studies was prepared in 14 steps from $S$ $(-)$-verbenone by adapting the synthetic procedures reported by Kato et al. ${ }^{12 a}$

\section{An unexpected cyclization of fuscol}

Upon treatment of 5 with glycoside 11 at $23{ }^{\circ} \mathrm{C}$ using the standard Yu glycosylation conditions (Table 1, entry 1), we observed a complex mixture of products, which co-eluted as a single, apolar band by normal-phase chromatography. Using reversedphase chromatography, we unexpectedly isolated small quantities of the xishacorenes along with several unidentifiable decomposition products. Upon lowering the reaction temperature to $-20{ }^{\circ} \mathrm{C}$, xishacorenes $\mathrm{A}-\mathrm{C}(\mathbf{1}, \mathbf{2}$, and 3$)$, as well as the previously unreported diastereomer $\mathbf{4}$, were isolated (Table $\mathbf{1}$, entry 2) in appreciable yields, with 3 as the major product. Following the screening of various acid conditions (Table 1, entries 5-10), we found the use of $\mathrm{BF}_{3} \cdot \mathrm{OEt}_{2}$ at $-78{ }^{\circ} \mathrm{C}$ to be optimal, giving the xishacorenes in a combined $49 \%$ isolated yield after 15 minutes (Table 1, entry 8). Intriguingly, cyclization was not observed using the triflimide gold complex in the absence of glycoside $\mathbf{1 1},{ }^{16}$ nor did the desired cyclization occur with bis(trifluoromethane)sulfonimide $\left(\mathrm{Tf}_{2} \mathrm{NH}\right)$ alone. ${ }^{17}$

\section{Analysis of the physical properties of the xishacorenes}

Following our preparation of $\mathbf{1 - 4}$, we sought to verify their reported physical properties. The originally reported molar extinction coefficient $(\varepsilon)$ values for each of the xishacorenes varied significantly (by factors of 10-20), which was surprising given their close structural similarity. Likewise, the reported $\varepsilon$ value for xishacorene $B$ is unexpectedly high $\left(147910 \mathrm{M}^{-1} \mathrm{~cm}^{-1}\right)$, and is more characteristic of a highly conjugated $\pi$ system. ${ }^{18}$ As $\varepsilon$ values are particularly important for the accurate determination of solution concentrations for biological studies, we remeasured these values in cyclohexane before pursuing bioactivity assays. We found the corresponding $\varepsilon$ values to equal $9448,11145,18594$, and $9721 \mathrm{M}^{-1} \mathrm{~cm}^{-1}$ for 1 , 2,3 , and 4 respectively. We also measured $\lambda_{\max }$ values for these compounds and obtained values consistent with what would be anticipated for these molecules $(239-240 \mathrm{~nm}$ for 1-4 in cyclohexane). ${ }^{19}$ We hypothesize that the inconsistencies between the previously reported values and those we measured may arise from the insolubility of these secondary metabolites in methanol, which had been used as the solvent in the original report. 


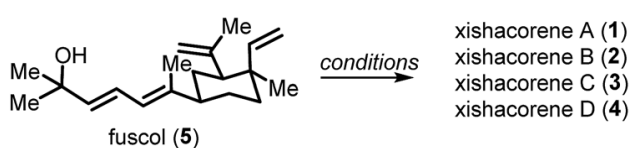

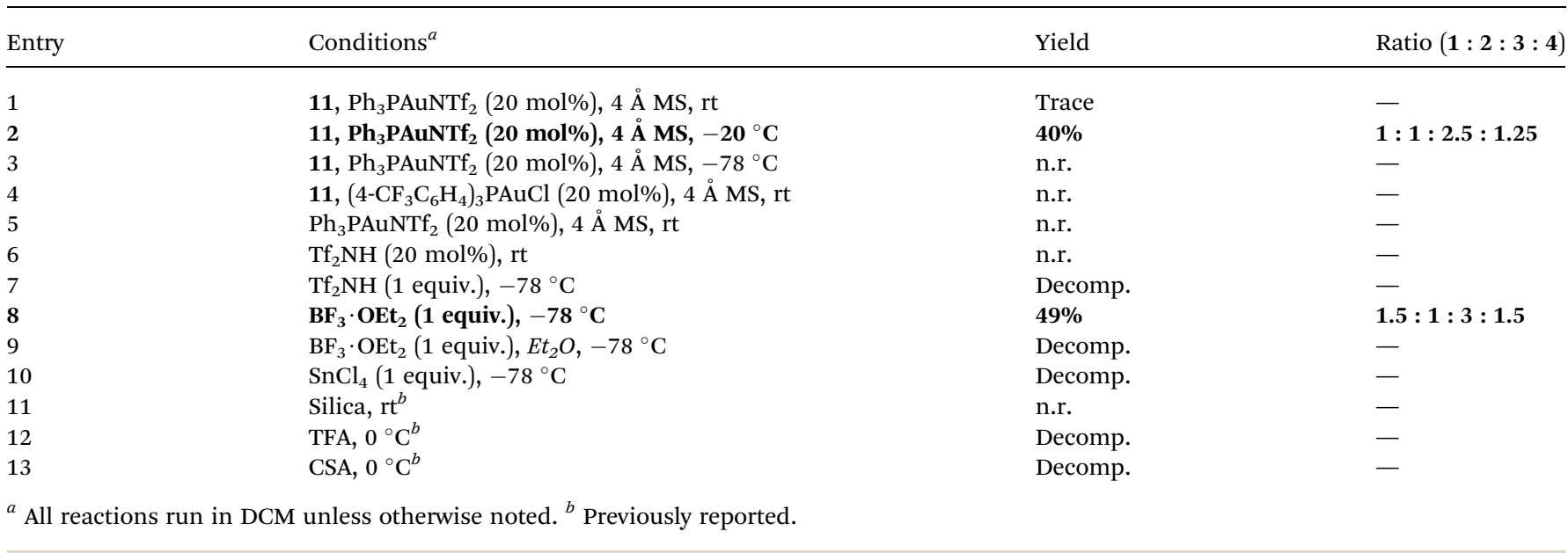

A lack of complete dissolution of the xishacorenes could explain the significant variations of the reported values from those we measured. Similarly, as noted in our previous report, ${ }^{\mathbf{1 1}}$ our measured optical rotation of synthetic xishacorene B differed from that reported for the naturally isolated material $\left([\alpha]_{\mathrm{D}}^{20}\right.$ of -11.3 reported, whereas we had found it to be -19.5 measured). We observed similar deviations for $\mathbf{1}$ and $\mathbf{3}$ as well and have provided updated $[\alpha]_{\mathrm{D}}^{20}$ values obtained using $\mathrm{CHCl}_{3}$ as the solvent (see the ESI $\dagger$ ). ${ }^{20}$

\section{Preliminary biological assays}

With the revised analytical properties of the xishacorenes determined, we undertook a series of biological assays to ascertain their bioactivity. Initial cell viability studies using THP1-Dual ${ }^{\mathrm{TM}}$ reporter cells with each of the assayed compounds confirmed that the majority of cells survived at 100 $\mu \mathrm{M}$, whereas we observed dramatic cell death at $1 \mathrm{mM}$, as determined by a CellTiter-Glo® assay (Fig. 3A). In order to determine the immunogenic activity of the xishacorenes, THP-

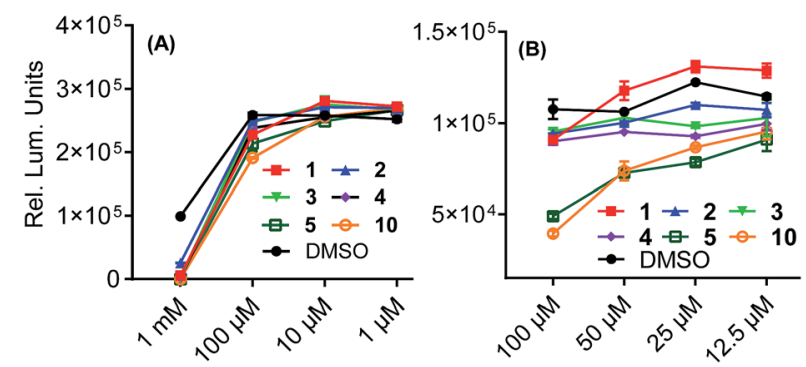

Fig. 3 THP-1 cell assay of compounds $1-5$ and 10 after $24 \mathrm{~h}$ of incubation. (A) CellTiter-Glo ${ }^{\circledR}$ assay measure of cell viability at each concentration. (B) Activity assay of compounds following stimulation with ML RR-S2 cGAMP (STING Promoter).
1-Dual ${ }^{\mathrm{TM}}$ cells featuring a secreted luciferase reporter to monitor activity of the IRF pathway were used. Each of the assayed compounds was dissolved in DMSO to create stock solutions. These solutions were then added to the media prior to incubation with or without a known STING (stimulator of interferon genes) agonist (ML RR-S2 cGAMP). ${ }^{21}$ After 24 hours of incubation, none of the assayed compounds emerged as antagonists of the IRF pathway, as monitored by luciferase expression. However, fuscol and hydroxylated xishacorene B derivative 10 were found to inhibit IRF activity induced by ML RR-S2 cGAMP (Fig. 3B). These observations suggest that the hydrophilic nature of fuscol and the fuscol-like analogue, unlike the highly apolar xishacorenes, is key to potency. Our future studies will seek to prepare other oxygenated variants of the xishacorenes that may have enhanced hydrophilicity.

\section{Conclusions}

In conclusion, we have demonstrated that fuscol undergoes an acid-mediated cyclization to produce the xishacorenes, supporting the proposed biosynthesis of this unique family of diterpenoids. Our isolation of xishacorene D also raises the possibility that this compound might co-occur alongside the other xishacorene congeners and may have been missed in the original isolation, since we obtained xishacorene $\mathrm{D}$ in a very small amount from the cyclization of fuscol. Bioactivity studies of fuscol and the xishacorenes, facilitated by a redetermination of the physical properties of the latter compounds, have provided unique insight into the importance of hydrophilic substituents to their bioactivity.

\section{Conflicts of interest}

WD and CN are employees and shareholders of Aduro Biotech, Inc. 


\section{Acknowledgements}

This work was supported by the National Science Foundation (NSF, CHE-1566430 to R. S.). Aduro Biotech is kindly acknowledged for a Postdoctoral Fellowship for A. R. R. The authors are grateful to Ken Metchette (Aduro Biotech) for UV-Vis data acquisition. We thank Dr Hasan Celik and Dr Jeffrey Pelton (UC Berkeley) for assistance with NMR experiments, and Dr Ulla Anderson for assistance with gathering HRMS data.

\section{Notes and references}

1 A. G. M. Barrett, T.-K. Ma and T. Mies, Synthesis, 2019, 51, 6782.

2 R. A. Yoder and J. N. Johnston, Chem. Rev., 2005, 105, 47304756.

3 F. Ye, Z. D. Zhu, J. S. Chen, J. Li, Y. C. Gu, W. L. Zhu, X. W. Li and Y. W. Guo, Org. Lett., 2017, 19, 4183-4186.

4 Y. Gopichand and F. J. Schmitz, Tetrahedron Lett., 1978, 39, 3641-3644.

5 The limited attempts to effect such a transformation in a laboratory setting by Guo, Li, and coworkers (see ref. 3), who isolated the xishacorenes, were not successful and resulted only in non-specific decomposition.

6 M. B. Saleh and R. G. Kerr, Aust. J. Chem., 2010, 63, 901-906.

7 (a) J. H. Shin and W. Fenical, J. Org. Chem., 1991, 56, 31533158; (b) E. Reina, C. Puentes, J. Rojas, J. Garcia, F. A. Ramos, L. Castellanos, M. Aragon and L. F. Ospina, Bioorg. Med. Chem. Lett., 2011, 21, 5888-5891.

8 (a) H. Miyaoka and Y. Yamada, Bull. Chem. Soc. Jpn., 2002, 75, 203-222; (b) Y. Gonzalez, D. Torres-Mendoza, G. E. Jones and P. L. Fernandez, Mediators Inflammation, 2015, 263543.

9 (a) P. B. Jacobson and R. S. Jacobs, J. Pharmacol. Exp. Ther., 1992, 262, 874-882; (b) P. B. Jacobson and R. S. Jacobs, J. Pharmacol. Exp. Ther., 1992, 262, 866-873; (c) P. B. Jacobson and R. S. Jacobs, FASEB J., 1991, 5, A510.

10 (a) M. Weber, K. Owens, A. Masarwa and R. Sarpong, Org. Lett., 2015, 17, 5432-5435; (b) A. Masarwa, M. Weber and R. Sarpong, J. Am. Chem. Soc., 2015, 137, 6327-6334; (c) Y. Kuroda, K. J. Nicacio, I. A. da Silva Jr, P. R. Leger, S. Chang, J. R. Gubiani, V. M. Deflon, N. Nagashima, A. Rode, K. Blackford, A. G. Ferreira, L. D. Sette,
D. E. Williams, R. J. Andersen, S. Jancar, R. G. S. Berlinck and R. Sarpong, Nat. Chem., 2018, 10, 938-945; (d) P. Finkbeiner, K. Murai, M. Röpke and R. Sarpong, J. Am. Chem. Soc., 2017, 139, 11349-11352.

11 I. Kerschgens, A. R. Rovira and R. Sarpong, J. Am. Chem. Soc., 2018, 140, 9810-9813.

12 (a) H. Kosugi, O. Yamabe and M. Kato, J. Chem. Soc., Perkin Trans. 1, 1998, 217-221; (b) M. Iwashima, H. Nagaoka, K. Kobayashi and Y. Yamada, Tetrahedron Lett., 1992, 33(1), 81-82; (c) E. J. Corey, B. E. Roberts and B. R. Dixon, J. Am. Chem. Soc., 1995, 117, 193-196.

13 D. H. Marchbank and R. G. Kerr, Tetrahedron, 2011, 67, 3053-3061.

14 B. Yu, Acc. Chem. Res., 2018, 51, 507-516.

15 See $\mathrm{ESI} \dagger$ for synthetic scheme and procedures.

16 For conditions involving $\mathbf{1 1}$, (i.e. entry 2) this reaction may occur through the glycosylated species $\mathbf{8}$, with the sugar serving as a leaving group. We did not, however, detect the formation of 8 during our studies.

17 Note that entries 11-13 represent previous attempts from the original isolation report.

18 For example $(\log \varepsilon)$ values of organic compounds, see: E. Pretsch, P. Bühlmann and M. Badertscher, Structure determination of organic compounds, Springer, Berlin, 4th rev. and enl. edn, 2009, vol. xv, p. 433.

19 The original report provides a $\lambda_{\max }$ difference of $16 \mathrm{~nm}$ between xishacorenes $\mathrm{A}$ and $\mathrm{B}$, a surprisingly large shift when considering the two contain identical chromophoric functional groups. We believe the observed $\lambda_{\max }$ values align more closely to those predicted by Woodward-Feiser Rules as well.

20 Varied optical rotation values may be possible in $\mathrm{CHCl}_{3}$ due to the presence of electrophilic chlorine ions. See: A. G. Myers, M. Siu and F. Ren, J. Am. Chem. Soc., 2002, 124, 4230-4232. We have also included optical rotation values for 1-3 that were taken in methanol.

21 L. Corrales, L. H. Glickman, S. M. McWhirter, D. B. Kanne, K. E. Sivick, G. E. Katibah, S. R. Woo, E. Lemmens, T. Banda, J. J. Leong, K. Metchette, T. W. Dubensky and T. F. Gajewski, Cell Rep., 2015, 11, 1018-1030. 